\title{
ON THE GLOBAL DIMENSION OF FIXED RINGS
}

\author{
MARTIN LORENZ
}

(Communicated by Donald S. Passman)

Dedicated to the memory of I. N. Herstein

\begin{abstract}
Let $G$ be a finite group acting on a $k$-algebra $R$, and let $S=R^{G}$ denote the fixed subring of $R$. Our main interest is in the case where $|G|$ is not invertible in $R$. Instead, we assume that $R$ is flat over $S$ and that the trivial $k G$-module $k$ has a periodic projective resolution. (For a field $k$ of characteristic $p$, the latter condition holds precisely if the Sylow $p$-subgroups of $G$ are cyclic or generalized quaternion.) We use a periodicity result for Extgroups, established here in a more general setting that is independent of group actions, to estimate the global dimension of $S$ in this case.
\end{abstract}

\section{INTRODUCTION}

Let $G$ be a finite group acting by automorphisms on a $k$-algebra $R$ ( $k$ some commutative ring), and let $S=R^{G}$ denote the fixed subring of $R$. In this note, we are concerned with bounding the (right) global dimension of $S$ in terms of the global dimension of $R$ and other related data.

In case the global dimension of $R$ is at most 1 and $|G|$ is invertible in $R$, one knows that $\mathrm{r} \cdot \operatorname{gldim}(S) \leq \mathrm{r} \cdot \operatorname{gldim}(R)$, by results of Levitzki [L] and Bergman [Be]. The situation becomes worse for larger global dimensions and for $|G|^{-1} \notin R$. For example, if $R=k[x, y]$ is the polynomial ring over a field $k$ of characteristic $\neq 2$, and $G=C_{2}$ acts on $R$ by $x \mapsto-x, y \mapsto-y$, then $R$ has global dimension 2 whereas $S=k\left[x^{2}, x y, y^{2}\right]$ has infinite global dimension [R]. Also, taking $R$ to be the ring of $2 \times 2$-matrices over a field $k$ with $\operatorname{char} k=2$, and $G=\left\langle\left(\begin{array}{ll}1 & 1 \\ 0 & 1\end{array}\right)\right\rangle \cong C_{2}$ acting by conjugation on $R$, one obtains $S \cong k \oplus k \varepsilon$ with $\varepsilon^{2}=0$. So r. $\operatorname{gldim}(R)=0$, but r. $\operatorname{gldim}(S)=\infty$.

Nevertheless, if $|G|^{-1} \in R$, then one has the following estimate (see 2.2 below):

$$
\text { r. } \operatorname{gldim}(S) \leq \mathrm{r} \cdot \operatorname{gldim}(R)+\operatorname{pdim}\left(R_{S}\right) .
$$

Received by the editors October 25, 1988, and, in revised form, December 12, 1988. Presented at the AMS meeting at Loyola University, Chicago on May 20, 1989 (special session Non-commutative ring theory).

1980 Mathematics Subject Classification (1985 Revision). Primary 16A60, 16A72; Secondary $20 \mathrm{C} 05$.

The author's research was supported in part by a grant from the National Science Foundation. 
The aim of this article is to establish a similar estimate without the assumption on $|G|$. Our main result is as follows.

Theorem. Put $\bar{S}=S / \operatorname{Tr}(R)$, where $\operatorname{Tr}: R \rightarrow S$ is the usual trace map, and

$$
\rho=\mathrm{r} \cdot \operatorname{gldim}(R)+\operatorname{pdim}\left(R_{S}\right) \text { and } \sigma=\mathrm{r} \cdot \operatorname{gldim}(\bar{S})+\operatorname{pdim}\left(\bar{S}_{S}\right) .
$$

Assume that

(1) ${ }_{s} R$ is flat, and

(2) the trivial $k G$-module $k$ has a resolution $0 \rightarrow k \rightarrow X_{c} \rightarrow \cdots \rightarrow X_{1} \rightarrow$ $k \rightarrow 0$ with all $X_{i}$ projective over $k G$.

Then either r. $\operatorname{gldim}(S) \leq \max \{\rho, \sigma\}$ or $\operatorname{r} \cdot \operatorname{gldim}(S)=\infty$.

Assumption (2) here is satisfied if $|G|^{-1} \in k$ (see 2.3). More interestingly, if $k$ is a field of characteristic $p$, then (2) holds precisely if the Sylow $p$-subgroups of $G$ are cyclic or generalized quaternion. Thus the important special case of one automorphism of finite order acting on $R$ is covered by (2). For further examples, see 2.4 below. The precise meaning of (1) is less clear, but $R$ is known to be projective over $S$ in some cases that arise naturally (see 2.6).

The above result is a consequence of a more precise periodicity result for Ext-groups that holds in the situation of the above theorem (see Theorem 2.7). In $\S 1$, we establish such a periodicity result for Ext-groups in a more general abstract setting, independent of group actions. This result is then applied, in $\S 2$, to the case of fixed subrings.

\section{PERIODICITY FOR EXT}

1.1 Lemma. Let $S \rightarrow \bar{S}$ be a surjective ring homomorphism with kernel I. Let $M_{S}$ be an $S$-module with $M \cdot I^{n}=0$ for some $n \geq 1$. Then $\operatorname{pdim}\left(M_{S}\right) \leq$ r. $\operatorname{gldim}(S)+\operatorname{pdim}\left(\bar{S}_{S}\right)$.

Proof. Arguing by induction on $n$, one reduces to the case $n=1$, where the assertion is well known (e.g., [Ro, Theorem 9.32]).

\subsection{Lemma. Let $S$ be a ring and let}

$$
\mathbf{T}: 0 \rightarrow T_{c+1} \rightarrow T_{c} \rightarrow \cdots \rightarrow T_{1} \rightarrow T_{0} \rightarrow 0
$$

be a complex of right $S$-modules, with $c \geq 1$. Assume that

$$
\delta=\max _{0 \leq n \leq c+1}\left\{\operatorname{pdim} H_{n}(\mathbf{T})\right\} \quad \text { and } \tau=\max _{1 \leq m \leq c}\left\{\operatorname{pdim}\left(T_{m}\right)\right\}
$$

are both finite. Let $V_{S}$ be an $S$-module. Then there are homomorphisms

$$
f^{q}: \operatorname{Ext}_{S}^{q+c}\left(T_{0}, V\right) \rightarrow \operatorname{Ext}_{S}^{q}\left(T_{c+1}, V\right) \quad(q>\tau)
$$

such that $f^{q}$ is epi for $q>\max \{\tau, \delta\}$, and $f^{q}$ is an isomorphism for $q>$ $\max \{\tau, \delta+1\}$. 
Proof. We first introduce a number of (cochain-) complexes. Let $\mathbf{Q}=\left(Q^{i}\right)_{i \geq 0}$ be an injective resolution of $V_{S}$. So $H^{i}(\mathbf{Q})=0$ for $i>0$ and $H^{0}(\mathbf{Q}) \cong V$. Form the double complex

$$
\mathbf{B}=\mathbf{H o m}_{S}(\mathbf{T}, \mathbf{Q})=\left(B^{p, q}\right)_{p, q \geq 0}, \quad B^{p, q}=\operatorname{Hom}_{S}\left(T_{p}, Q^{q}\right)
$$

and its associated total complex

$$
\mathbf{C}=\operatorname{Tot}(\mathbf{B})=\left(C^{n}\right)_{n \geq 0}, \quad C^{n}=\bigoplus_{p+q=n} B^{p, q} .
$$

Note that $B^{p, q}=0$ for $p>c+1$ as $T_{p}=0$ in this case.

Step 1. $H^{n}(\mathbf{C})=0$ for $n>\delta+c+1$.

Proof. Since $\mathbf{Q}_{S}$ is injective, there is a Künneth spectral sequence $\left\{E_{r}\right\}$ with

$$
E_{2}^{p, q} \cong \bigoplus_{i+j=q} \operatorname{Ext}_{S}^{p}\left(H_{i}(\mathbf{T}), H^{j}(\mathbf{Q})\right) \underset{p}{\Rightarrow} H^{p+q}(\mathbf{C})
$$

(see [Ro, Theorem 11.34] or [G, Theorem 5.4.1]). Thus

$$
E_{2}^{p, q}=\operatorname{Ext}_{S}^{p}\left(H_{q}(\mathbf{T}), V\right)=0
$$

if either $q>c+1$ or $p>\delta$. Therefore, $H^{p+q}(\mathbf{C})=0$ if $p+9>\delta+c+1$.

Step 2. The maps $f^{q}$.

Consider the first filtration of $\mathbf{C}$, as in [C-E, p. 330] (omitting the subscript I):

$$
\left(F^{p} \mathbf{C}\right)^{n}=\bigoplus_{\substack{r \geq p \\ r+s=n}} B^{r, s}
$$

The corresponding spectral sequence $\left\{E_{r}^{\prime}\right\}$ converges to $H^{*}(\mathbf{C})$ and has $E_{2}^{\prime}$ term (notation as in [C-E, pp. 330-331])

$$
E_{2}^{\prime} \cong H_{\mathrm{I}} H_{\mathrm{II}}(\mathbf{B}) \text {, }
$$

where

$$
H_{\mathrm{II}}^{p, q}(\mathbf{B})=H^{q}\left(B^{p, *}\right)=H^{q}\left(\operatorname{Hom}_{S}\left(T_{p}, \mathbf{Q}\right)\right)=\operatorname{Ext}_{S}^{q}\left(T_{p}, V\right) .
$$

In particular, since $T_{p}=0$ for $p>c+1$, we have $H_{\mathrm{II}}^{p, *}(\mathbf{B})=0$ for $p>c+1$ and so $\left(E_{2}^{\prime}\right)^{p, q}=0$ for $p>c+1$. Since the differential $d_{r}$ of $E_{r}^{\prime}$ has bidegree $(r, 1-r)$, it follows that $d_{c+2}=0$ and so

$$
E_{\infty}^{\prime}=E_{c+2}^{\prime} \text {. }
$$

Moreover, by definition of $\tau, H_{\mathrm{II}}^{p, q}(\mathbf{B})=0$ for $q>\tau$ and $p \neq 0, c+1$. Consequently, for $q>\tau$, we have

$$
\left(E_{2}^{\prime}\right)^{p, q}=\left\{\begin{array}{ll}
0 & \text { if } p \neq 0, c+1 \\
\operatorname{Ext}_{S}^{q}\left(T_{0}, V\right) & \text { if } p=0 \\
\operatorname{Ext}_{S}^{q}\left(T_{c+1}, V\right) & \text { if } p=c+1
\end{array} \quad(q>\tau)\right.
$$


By considering the bidegree of the differential $d_{r}$ of $E_{r}^{\prime}$, one sees that

$$
\begin{aligned}
q \geq \tau+c & \Rightarrow d_{r}^{0, q}=0 \text { for } 2 \leq r \leq c \\
& \Rightarrow\left(E_{2}^{\prime}\right)^{0, q}=\left(E_{3}^{\prime}\right)^{0, q}=\cdots=\left(E_{c+1}^{\prime}\right)^{0, q}
\end{aligned}
$$

and

$$
\begin{aligned}
q \leq \tau & \Rightarrow d_{r}^{c+1-r, q-(1-r)}=0 \text { for } 2 \leq r \leq c \\
& \Rightarrow\left(E_{2}^{\prime}\right)^{c+1, q}=\left(E_{3}^{\prime}\right)^{c+1, q}=\cdots=\left(E_{c+1}^{\prime}\right)^{c+1, q} .
\end{aligned}
$$

Therefore, for $q \geq \tau$, the differential $d_{c+1}$ yields a homomorphism

$$
\left(E_{2}^{\prime}\right)^{0, q+c} \stackrel{\sim}{\longrightarrow}\left(E_{c+1}^{\prime}\right)^{0, q+c} \stackrel{d_{c+1}}{\longrightarrow}\left(E_{c+1}^{\prime}\right)^{c+1, q} \stackrel{\sim}{\longrightarrow}\left(E_{2}^{\prime}\right)^{c+1, q} .
$$

For $q>\tau$, this is the required homomorphism

$$
f^{q}: \operatorname{Ext}_{S}^{q+c}\left(T_{0}, V\right) \rightarrow \operatorname{Ext}_{S}^{q}\left(T_{c+1}, V\right) .
$$

Step 3. Surjectivity and injectivity.

Since $H^{n}(\mathbf{C})=0$ for $n>\delta+c+1$, by Step 1, we know that $\left(E_{\infty}^{\prime}\right)^{p, q}=0$ for $p+q>\delta+c+1$. In particular,

$$
0=\left(E_{\infty}^{\prime}\right)^{c+1, q}=\left(E_{c+2}^{\prime}\right)^{c+1, q}=\left(E_{c+1}^{\prime}\right)^{c+1, q} / d_{c+1}\left(E_{c+1}^{\prime}\right)^{0, q+c}
$$

for $c+1+q>\delta+c+1$, and so $f^{q}$ is surjective for $q>\delta, q>\tau$. Also,

$$
0=\left(E_{\infty}^{\prime}\right)^{0, q+c}=\left(E_{c+2}^{\prime}\right)^{0, q+c}=\operatorname{Ker}\left(\left.d_{c+1}\right|_{\left(E_{c+1}^{\prime}\right)^{0, q+c}}\right) .
$$

for $q+c>\delta+c+1$. Thus $f^{q}$ is injective for $q>\delta+1, q>\tau$. This proves the lemma.

1.3 Proposition. Let $S \subseteq R$ be an inclusion of rings and let $S \rightarrow \bar{S}$ be a surjective ring homomorphism. Assume that

$$
\rho=\mathrm{r} \cdot \operatorname{gldim}(R)+\operatorname{pdim}\left(R_{S}\right) \text { and } \sigma=\mathrm{r} \cdot \operatorname{gldim}(\bar{S})+\operatorname{pdim}\left(\bar{S}_{S}\right)
$$

are both finite. Suppose that there is a complex of $(S, S)$-bimodules

$$
\mathbf{P :} 0 \rightarrow P_{c+1} \rightarrow P_{c} \rightarrow \cdots \rightarrow P_{1} \rightarrow P_{0} \rightarrow 0
$$

with $c \geq 1$, such that

(1) each ${ }_{S} P_{i}$ is flat;

(2) for $1 \leq i \leq c, P_{i}$ is an $(S, S)$-bimodule direct summand of some $(S, R)$ bimodule;

(3) the right $S$-action on $H_{*}(\mathbf{P})$ factors through $\bar{S}$.

Then, for any two $S$-modules $V_{S}$ and $W_{S}$, there exist homomorphisms

$$
f^{q}: \mathrm{Ext}_{S}^{q+c}\left(W \otimes_{S} P_{0}, V\right) \rightarrow \operatorname{Ext}_{S}^{q}\left(W \otimes_{S} P_{c+1}, V\right) \quad(q>\rho)
$$

such that $f^{q}$ is epi for $q>\max \{\rho, \sigma\}$ and an isomorphism for $q>\max \{\rho, \sigma+1\}$.

Proof. Put $\mathbf{T}=W \otimes_{S} \mathbf{P}$, a complex of right $S$-modules. We estimate the numbers $\delta$ and $\tau$ in Lemma 1.2. First, by assumption (2), $T_{i}$ is an $S$-module 
direct summand of some right $R$-module, say $Q_{i}(i=1, \ldots, c)$. Therefore, by [Ro, Theorem 9.32],

$$
\operatorname{pdim}\left(T_{i}\right)_{S} \leq \operatorname{pdim}\left(Q_{i}\right)_{S} \leq \operatorname{pdim}\left(Q_{i}\right)_{R}+\operatorname{pdim}\left(R_{S}\right) \leq \rho
$$

Hence

$$
\tau \leq \rho \text {. }
$$

Next, we claim that $H_{n}(\mathbf{T}) \cdot I^{n+1}=0$ for $0 \leq n \leq c+1$, where $I$ denotes the kernel of the map $S \rightarrow \bar{S}$. Indeed, since ${ }_{S} \mathbf{P}$ is flat, by (1), there is a Künneth spectral sequence $\left\{E^{r}\right\}$ with

$$
E_{p, q}^{2} \cong \operatorname{Tor}_{p}^{S}\left(W, H_{q}(\mathbf{P})\right) \underset{p}{\Rightarrow} H_{p+q}(\mathbf{T})
$$

([Ro, Theorem 11.34] or [M, Chapter XII, Theorem 12.1]). By (3), we have $E_{p, q}^{2} \cdot I=0$. The spectral sequence yields a chain

$$
0=F^{-1} H_{n} \subseteq F^{0} H_{n} \subseteq \cdots \subseteq F^{n} H_{n}=H_{n}
$$

of $S$-submodules of $H_{n}=H_{n}(\mathrm{~T})$ such that each $F^{p} H_{n} / F^{p-1} H_{n}$ is isomorphic to a subquotient of $E_{p, n-p}^{2}$. Therefore, $H_{n}(\mathrm{~T}) \cdot I^{n+1}=0$, as we have claimed.

Lemma 1.1 now yields $\delta \leq \sigma$, and so the proposition follows from Lemma 1.2.

1.4 Remarks. The proof of Proposition 1.3 works without essential changes if (3) is weakened to

$\left(3^{\prime}\right)$ Some power of $I=\operatorname{Ker}(S \rightarrow \bar{S})$ annihilates each $H_{n}(\mathbf{P})$.

Moreover, arguing somewhat differently (without invoking the spectral sequence) in the last part of the proof, the conclusion of Proposition 1.3 still holds if (1) is replaced by

$\left(1^{\prime}\right)$ Some power of $I$ annihilates each $\operatorname{Tor}_{i}^{S}\left(W, P_{j}\right)$, for all $i>0$.

However, these generalizations don't seem to be useful, at least not for our applications to fixed rings in $\S 2$.

1.5 Corollary. In the situation of Proposition 1.3, assume in addition that $S$ is an $(S, S)$-bimodule direct summand of $P_{c+1}$. Then, for any $S$-module $V_{S}$, either $\operatorname{idim}\left(V_{S}\right) \leq \max \{\rho, \sigma\}$ or $\operatorname{idim}\left(V_{S}\right)=\infty$. Consequently, either $r \cdot \operatorname{gldim}(S) \leq$ $\max \{\rho, \sigma\}$ or r. $\operatorname{gldim}(S)=\infty$.

Proof. Assume that $\operatorname{idim}\left(V_{S}\right)>\max \{\rho, \sigma\}$, so $\operatorname{Ext}_{S}^{q}(W, V) \neq 0$ for some $S$ module $W_{S}$ and some $q>\max \{\rho, \sigma\}$. Proposition 1.3 yields an epimorphism $\operatorname{Ext}_{S}^{q+c}\left(W \otimes_{S} P_{0}, V\right) \rightarrow \operatorname{Ext}_{S}^{q}\left(W \otimes_{S} P_{c+1}, V\right)$ and the latter group is nonzero, since it maps onto $\operatorname{Ext}_{S}^{q}(W, V)$, by assumption on $P_{c+1}$. Therefore,

$$
\operatorname{Ext}_{S}^{q+c}\left(W \otimes_{S} P_{0}, V\right) \neq 0,
$$

and we can continue with $W \otimes_{S} P_{0}$ in place of $W$. 


\section{Periodic ReSOLUTIONS FOR GROUPS}

2.1 Notations. The following notations will be kept throughout this section. $R$ will be an algebra over some commutative ring $k$, and $G$ will be a finite group acting on $R$ by $k$-algebra automorphisms that will be written $r \mapsto r^{g}(r \in$ $R, g \in G)$. We let $S=R^{G}$ denote the fixed subring of $R$. As usual, $\operatorname{Tr}: R \rightarrow S$ is the trace map given by $\operatorname{Tr}(r)=\sum_{g \in G} r^{g}(r \in R)$. Since $\operatorname{Tr}$ is an $(S, S)$ bimodule map, its image $\operatorname{Tr}(R)$ is an ideal of $S$. We put $\bar{S}=S / \operatorname{Tr}(R)$. The augmentation ideal of the group algebra $k G$ will be denoted by $\omega G$, and we put $\widehat{G}=\sum_{g \in G} g \in k G$. Viewing $R$ as a right $k G$-module via the given $G$-action, the trace map can be written as $\operatorname{Tr}(r)=r^{\widehat{G}}(r \in R)$.

2.2 The case $|G|^{-1} \in R$. If $|G|^{-1} \in R$, then the map $|G|^{-1} \cdot \operatorname{Tr}: R \rightarrow S$ is the identity on $S$. thus $S$ is an $(S, S)$-bimodule direct summand of $R$, and [K, Theorem 5 on p. 173] implies

$$
\text { r. } \operatorname{gldim}(S) \leq \mathrm{r} \cdot \operatorname{gldim}(R)+\operatorname{pdim}\left(R_{S}\right) .
$$

Thus, in the following, our emphasis will be on the case where $|G|^{-1} \notin R$, although the case $|G|^{-1} \in R$ is covered by the following, too.

2.3 Periodic resolutions. Following [A], we say that the trivial $k G$-module $k$ is periodic if there exists an exact sequence of left (say) $k G$-modules

$$
\mathbf{X}: 0 \rightarrow k \stackrel{\mu}{\longrightarrow} X_{c} \stackrel{\phi_{c}}{\longrightarrow} \cdots \rightarrow X_{i} \stackrel{\phi_{i}}{\longrightarrow} \cdots \rightarrow X_{1} \stackrel{\varepsilon}{\longrightarrow} k \rightarrow 0,
$$

where all $X_{i}$ are projective over $k G$. The smallest such $c$ is called the period of $k$. (In [A], these concepts are introduced, in the same way, for arbitrary $k G$ modules.) The case where $k$ is projective over $k G$ or, equivalently, $|G|^{-1} \in k$, is included here via the obvious sequence $0 \rightarrow k \rightarrow X_{1}=k \oplus k \rightarrow k \rightarrow 0$.

2.4 Remarks and Examples. (a) If $k$ is periodic with period $c$, then the (Tate) cohomology ring $\widehat{H}^{*}(G, k)$ has an invertible element $u \in \widehat{H}^{c}(G, k)$. In particular, using the fact that $\widehat{H}^{*}(G, k)$ is anticommutative, one sees that, if $2 \neq 0$ in $\widehat{H}^{0}(G, k)=k /|G| k$, then $c$ must be even. But, of course, the case of odd $c$ also occurs, e.g. $c=1$ when $|G|=2$ and $2=0$ in $k$.

(b) If $\mathbf{Z}$ is periodic for $\mathbf{Z} G$, then any $k$ is periodic for $k G$. In this case, all abelian subgroups of $G$ must be cyclic ([Br, Theorem 9.5 on p. 157]). All finite groups of this type have been classified by Zassenhaus [Z] in the solvable case (see also [W, Theorem 6.1.11]) and by Suzuki [S] in general.

A construction of periodic free $\mathbf{Z} G$-resolutions $\mathbf{X}$ for $\mathbf{Z}$ starting with a fixed-point-free complex representation of $G$ (that is, a finite-dimensional $\mathbf{C} G$ module $V$ such that $\{v \in V \mid g v=v\}=0$ holds for all $1 \neq g \in G$ ) is described in [Br, p. 154]. The complete classification of all groups admitting a fixed-pointfree representation can be found in [W, Theorems 6.1.11 and 6.3.1]. These 
groups include in particular the finite subgroups of the multiplicative group of the quaternions $\mathbf{H}$ :

-the finite cyclic groups,

- the generalized quaternion groups $Q_{4 m}=\left\langle x, y \mid y^{2}=x^{m}, x y x=y\right\rangle$ $(m \geq 2)$,

- the binary tetrahedral group (order 24), the binary octahedral group (order 48 ), and the binary icosahedral group $S L(2,5)$ (order 120).

For further examples and more details see [Br, pp. 154-156].

(c) If $k$ is a field of characteristic $p>0$, then the trivial $k G$-module $k$ is periodic if and only if all elementary abelian $p$-subgroups of $G$ are cyclic (see [A-E]). The latter condition is satisfied if and only if the Sylow $p$-subgroups of $G$ are either cyclic or generalized quaterion (for $p=2$ only) (see [Br, p. 157]).

2.5 Lemma. Assume that the trivial $k G$-module $k$ is periodic with period $c$. Then there exists a complex of $(S, S)$-bimodules

$$
\mathbf{P}: 0 \rightarrow P_{c+1} \rightarrow P_{c} \rightarrow \cdots \rightarrow P_{1} \rightarrow P_{0} \rightarrow 0
$$

such that

(1) $P_{0} \cong S \cong P_{c+1}$

(2) for $1 \leq i \leq c, P_{i}$ is an $(S, S)$-bimodule direct summand of some $R^{\left(\alpha_{i}\right)}$;

(3) the left and right $S$-actions on $H_{*}(\mathbf{P})$ factor through $\bar{S}$.

Proof. Let

$$
\mathbf{X}: 0 \rightarrow k \stackrel{\mu}{\longrightarrow} X_{c} \stackrel{\phi_{c}}{\longrightarrow} \ldots \stackrel{\phi_{2}}{\longrightarrow} X_{1} \stackrel{\varepsilon}{\longrightarrow} k \rightarrow 0
$$

be a periodic $k G$-resolution as in 2.3. Expand $\mathbf{X}$ into a complete periodic resolution

$$
\widehat{\mathbf{X}}: \cdots \rightarrow X_{c+1}=X_{1} \stackrel{\phi_{c+1}=\mu \circ \varepsilon}{\longrightarrow} X_{c} \stackrel{\phi_{c}}{\longrightarrow} \cdots \stackrel{\phi_{2}}{\longrightarrow} X_{1} \stackrel{\phi_{1}=\mu \circ \varepsilon}{\longrightarrow} X_{0}=X_{c} \rightarrow \cdots .
$$

Consider the complex $\mathbf{Q}=R \otimes_{k G} \widehat{\mathbf{x}}$, with maps $\psi_{i}=\operatorname{id}_{R} \otimes \phi_{i}$. The $(S, S)$ bimodule structure on $R$ makes $\mathbf{Q}$ a complex of $(S, S)$-bimodules. Moreover, since each $X_{i}$ is a direct summand of some $k G^{\left(\alpha_{i}\right)}, Q_{i}=R \otimes_{k G} X_{i}$ is an $(S, S)$-bimodule direct summand of $R \otimes_{k G} k G^{\left(\alpha_{i}\right)}=R^{\left(\alpha_{i}\right)}$. The homology of $\mathbf{Q}$ is given by

$$
H_{i}(\mathbf{Q})=\widehat{H}_{i-1}(G, R),
$$

the Tate homology of $G$ with coefficients in $R$, up to an index shift. The $(S, S)$-bimodule structure on $H_{*}(\mathbf{Q})=\widehat{H}_{*-1}(G, R)$ can be viewed as the (left and right) cap product action of $S=H^{0}(G, R)^{\prime}$ on $\widehat{H}_{*}(G, R)$, and this action factors through $\bar{S}=\widehat{H}^{0}(G, R)$.

We now modify $\mathbf{X}$ and $\mathbf{Q}$ to obtain $\mathbf{P}$. Note that $\operatorname{Im}(\mu) \subseteq \operatorname{ann}_{X_{c}}(\omega G)=$ $\widehat{G} \cdot X_{c}$ and so, identifying $k$ with $k \cdot \widehat{G} \subseteq k G$, we have $\mu(\widehat{G})=\widehat{G} \cdot \xi$ for some $\xi \in X_{c}$. Define a $k G$-linear map $\mu^{\prime}: k G \rightarrow X_{c}$ by $\mu^{\prime}(1)=\xi$, so $\left.\mu^{\prime}\right|_{k \widehat{G}}=\mu$. Therefore, $\left.\phi_{c} \circ \mu^{\prime}\right|_{k \widehat{G}}=0$, and hence $\operatorname{Im}\left(\phi_{c} \circ \mu^{\prime}\right) \subseteq \operatorname{ann}_{X_{c}}(\widehat{G})=(\omega G) \circ X_{c-1}$. 
Furthermore, define $\varepsilon^{\prime}: X_{1} \rightarrow k G$ to be the composite $X_{1} \stackrel{\varepsilon}{\longrightarrow} k \cong k \cdot \widehat{G} \hookrightarrow k G$. Note that $\phi_{1}: X_{1} \rightarrow X_{0}=X_{c}$ factors as $f_{1}=\mu^{\prime} \circ \varepsilon^{\prime}$.

Now define $(S, S)$-bimodule maps $\tilde{\mu}: R=R \otimes_{k G} k G \rightarrow Q_{c}=R \otimes_{k G} X_{c}$ by $\tilde{\mu}=\mathrm{id}_{R} \otimes \mu^{\prime}$ and $\tilde{\varepsilon}: Q_{1}=R \otimes_{k G} X_{1} \rightarrow R=R \otimes_{k G} k G$ by $\tilde{\varepsilon}=\mathrm{id}_{R} \otimes \varepsilon^{\prime}$. Then the image of $\tilde{\varepsilon}$ is equal to the image of $R \otimes_{k G} k \widehat{G} \rightarrow R \otimes_{k G} k G=R$, that is

$$
\tilde{\varepsilon}\left(Q_{1}\right)=\operatorname{Tr}(R) \text {. }
$$

Next, we show that $\tilde{\mu}$ is mono on $\operatorname{Tr}(R) \subseteq R$. To see this, embed $X_{c}$ as a direct summand in a free module $F=k G^{(I)}$, with corresponding map $\lambda: X_{c} \rightarrow$ $F$. Then, putting $\phi=\lambda \circ \mu^{\prime}: k G \rightarrow F$ and $\widetilde{\phi}=\mathrm{id}_{R} \otimes \phi: R \rightarrow R \otimes_{k G} F \cong R^{(I)}$, we have $\operatorname{Ker}(\widetilde{\mu})=\operatorname{Ker}(\widetilde{\phi})$. The map

$$
\left.\phi\right|_{k \widehat{G}}: k \widehat{G} \stackrel{\mu}{\longrightarrow} \widehat{G} \cdot X_{c} \stackrel{\lambda}{\longrightarrow} \widehat{G} \cdot F=(\widehat{G} k)^{(I)}
$$

is $k$-split, since $\mathbf{X}$ splits over $k$, and hence $\mu$ does. Therefore, $\phi(\widehat{G})=\left(\widehat{G} \xi_{i}\right)_{i \in I}$ for certain $\xi_{i} \in k$ (almost all 0 ) with $\sum_{i} \xi_{i} \tau_{i}=1$ for suitable $\tau_{i} \in k$. Now, for any $r^{\widehat{G}} \in \operatorname{Tr}(R)$, we have in $R \otimes_{k G} F=R^{(I)}$,

$$
\begin{aligned}
\widetilde{\phi}\left(r^{\widehat{G}}\right) & =r^{\widehat{G}} \otimes \phi(1)=r \otimes \phi(\widehat{G}) \\
& =r \otimes\left(\widehat{G} \xi_{i}\right)_{i \in I}=\left(r^{\widehat{G}} \xi_{i}\right)_{i \in I},
\end{aligned}
$$

and so, if $\tilde{\phi}\left(r^{\widehat{G}}\right)=0$, then $r^{\widehat{G}}=\sum_{i} r^{\widehat{G}} \xi_{i} \tau_{i}=0$, as we have claimed. It follows that $\operatorname{Tr}(R) \cdot \operatorname{Ker}\left(\left.\widetilde{\mu}\right|_{S}\right)=0=\operatorname{Ker}\left(\left.\widetilde{\mu}\right|_{S}\right) \cdot \operatorname{Tr}(R)$.

Finally, we claim that $\widetilde{\mu}(S) \subseteq \operatorname{Ker}\left(\psi_{c}\right)$. Indeed, since

$$
\operatorname{Im}\left(\phi_{c} \circ \mu^{\prime}\right) \subseteq(\omega G) \cdot X_{c-1},
$$

it follows that $\psi_{c} \circ \tilde{\mu}(S)$ is contained in the canonical image of $S \otimes_{k G}(\omega G) X_{c-1}$ in $Q_{c-1}=R \otimes_{k G} X_{c-1}$, and this image equals 0 , since $S^{\omega G}=0$. Thus we have the following complex of $(S, S)$-bimodules

$$
\mathbf{P}: 0 \rightarrow P_{c+1}=S \stackrel{\left.\widetilde{\mu}\right|_{S}}{\longrightarrow} P_{c}=Q_{c} \stackrel{\psi_{c}}{\longrightarrow} \cdots \stackrel{\psi_{2}}{\longrightarrow} P_{1}=Q_{1} \stackrel{\widetilde{\varepsilon}}{\rightarrow} P_{0}=S \rightarrow 0 .
$$

Its homology is: $H_{0}(\mathbf{P})=\bar{S}, H_{1}(\mathbf{P})=H_{1}(\mathbf{Q})$ (because $\psi_{1}=\tilde{\mu} \circ \widetilde{\varepsilon}$ and $\tilde{\mu}$ is injective on $\operatorname{Im}(\widetilde{\varepsilon})=\operatorname{Tr}(R))$, and $H_{i}(\mathbf{P})=H_{i}(\mathbf{Q}) \quad(i=1, \ldots, c-1)$. Moreover, $H_{c+1}(\mathbf{P})=\operatorname{Ker}\left(\left.\tilde{\mu}\right|_{S}\right)$ is annihilated, on both sides, by $\operatorname{Tr}(R)$ and $H_{c}(\mathbf{P})=$ $\operatorname{Ker}\left(\psi_{c}\right) / \widetilde{\mu}(S)$ is an image of $H_{c}(\mathbf{Q})=\operatorname{Ker}\left(\psi_{c}\right) / \psi_{1}\left(Q_{1}\right)=\operatorname{Ker}\left(\psi_{c}\right) / \widetilde{\mu}\left(\widetilde{\varepsilon}\left(Q_{1}\right)\right)=$ $\operatorname{Ker}\left(\psi_{c}\right) / \widetilde{\mu}(\operatorname{Tr}(R))$. Thus (3) is satisfied, and so $\mathbf{P}$ has all the required properties.

2.6 Flatness and projectivity. If the trivial $k G$-module $k$ is periodic, then Lemma 2.5 guarantees that the hypotheses of Proposition 1.3 are satisfied, except possibly for the flatness of all ${ }_{S} P_{i}$. For this, it suffices to assume that ${ }_{S} R$ is flat. I am not aware of any results specifically in this direction, but there 
do exist quite a few results ensuring projectivity of ${ }_{S} R$. For example, ${ }_{S} R$ is known to be projective in each of the following cases:

(a) If the skew group ring $T=R * G$ is a simple ring (it actually suffices to assume that $T=T \widehat{G} T$, where $\left.\widehat{G}=\sum_{g \in G} g \in T\right)$, then $R$ is finitely generated projective over $S$, on both sides (e.g., [Mo, proof of Theorem 2.4]). This happens, for example, if $R$ has no nontrivial $G$-invariant ideals and $G$ consists of outer automorphisms of $R$ (use [Mo, Lemma 3.16]).

(b) If $R$ is a finite direct product of simple rings and $|G|^{-1} \in R$, then $R$ is finitely generated projective over $S$ [H-R].

(c) Assume that $R$ is hereditary and $|G|^{-1} \in R$. Then each of the following implies that $R$ is projective over $S$, although not necessarily finitely generated in all cases: $R$ is semiprime Noetherian, $R$ is commutative von Neumann regular, $R$ is reduced von Neumann regular and $G$ is solvable [J].

2.7 Theorem. Let $R$ be an algebra over a commutative ring $k$ and let $G$ be a finite group of k-algebra automorphisms of $R$. Put $S=R^{G}, \bar{S}=S / \operatorname{Tr}(R)$ and assume that

$$
\rho=\mathrm{r} \cdot \operatorname{gldim}(R)+\operatorname{pdim}\left(R_{S}\right) \text { and } \sigma=\mathrm{r} \cdot \operatorname{gldim}(\bar{S})+\operatorname{pdim}\left(\bar{S}_{S}\right) .
$$

are both finite. Assume further that

(1) ${ }_{s} R$ is flat, and

(2) the trivial $k G$-module $k$ is periodic of period $c$.

Then, for any $S$-modules $V_{S}$ and $W_{S}$, there exist homomorphisms

$$
f^{q}: \operatorname{Ext}_{S}^{q+c}(W, V) \rightarrow \operatorname{Ext}_{S}^{q}(W, V) \quad(q>\rho)
$$

such that $f^{q}$ is epi for $q>\max \{\rho, \sigma\}$ and is an isomorphism for $q>$ $\max \{\rho, \sigma+1\}$. Consequently, either $\operatorname{r} \cdot \operatorname{gldim}(S) \leq \max \{\rho, \sigma\}$ or $\operatorname{r} \cdot \operatorname{gldim}(S)=$ $\infty$.

Proof. In view of the remarks in 2.6, this follows from Lemma 2.5 and Proposition 1.3.

2.8 The case $|G|=2$. We end this article by illustrating Theorem 2.7 in the special case where $|G|=2,2 k=0$, and the skew group ring $T=T * G$ is simple, or at least $T=T \widehat{G} T$. In this case, we have $\rho=\mathrm{r} \cdot \operatorname{gldim}(R)$ (see 2.6). Moreover, the exact sequence of $S$-modules

$$
0 \rightarrow S \stackrel{\text { incl. }}{\longrightarrow} R \stackrel{\mathrm{Tr}}{\longrightarrow} S \rightarrow \bar{S} \rightarrow 0
$$

shows that $\operatorname{pdim}\left(\bar{S}_{S}\right) \leq 2$. Therefore, $\sigma \leq \mathrm{r} \cdot \operatorname{gldim}(\bar{S})+2$. Theorem 2.7 now implies that

一either r. $\operatorname{gldim}(S)=\infty$

-or r. $\operatorname{gldim}(S) \leq \max \{$ r. $\operatorname{gldim}(R)$, r. $\operatorname{gldim}(\bar{S})+2\}$.

For a lower bound, we note that if $\operatorname{Tr}(R) \neq S$, then $\operatorname{pdim}(\bar{S}) \geq 2$, because otherwise $\operatorname{Tr}(R)$ would be projective over $S$, and hence $S$ would be an $S$ module direct summand of $R$. But the equality $T=T \widehat{G} T$ implies that $R=$ $R \cdot \operatorname{Tr}(R)$, which leads to a contradiction, since $\operatorname{Tr}(R) \neq S$. 


\section{ACKNOWLEDGMENT}

It is a pleasure to thank $\mathrm{K}$. Goodearl for his helpful comments on a preliminary version of this article.

\section{REFERENCES}

[A] J. L. Alperin, Periodicity in groups, Illinois J. Math. 21 (1977), 776-783.

[A-E] J. L. Alperin and L. Evens, Representations, resolutions and Quillen's dimension theorem, J. Pure Appl. Algebra 22 (1981), 1-9.

[B] G. Bergman, Groups acting on hereditary rings, Proc. London Math. Soc. 23 (1971), 70-82.

[Br] K. S. Brown, Cohomology of groups, Springer-Verlag, New York, 1982.

[C-E] H. Cartan and S. Eilenberg, Homological algebra, Princeton Univ. Press, Princeton, N. J., 1956.

[G] R. Godement, Topologie algébrique et théorie des faisceaux, 3rd ed., Hermann, Paris, 1973.

[H-R] D. Handelman and G. Renault, Actions of finite groups on selfinjective rings, Pacific J. Math. 89 (1980), 69-80.

[J] S. Jøndrup, When is the ring a projective module over the fixed point ring?, Comm. Algebra 16 (1988), 1971-1992.

[K] I. Kaplansky, Fields and rings, 2nd ed., Univ. Chicago Press, Chicago, Ill., 1972.

[L] J. Levitzki, On automorphisms of certain rings, Ann. of Math. 36 (1935), 984-992.

[M] S. MacLane, Homology, 3rd corr. printing, Springer-Verlag, Berlin, 1975.

[Mo] S. Montgomery, Fixed rings of finite automorphism groups of associative rings, Lecture Notes in Math., no. 818, Springer, Berlin, 1980.

[R] O. Riemenschneider, Deformationen von Quotientensingularitäten nach zyklischen Gruppen, Math. Ann. 209 (1974), 211-248.

[Ro] J. Rotman, An introduction to homological algebra, Academic Press, Orlando, 1979.

[W] J. A. Wolf, Spaces of constant curvature, 5th ed., Publish or Perish, Wilmington, 1984.

[S] M. Suzuki, On finite groups with cyclic Sylow subgroups for all odd primes, Amer. J. Math. 77 (1955), 657-691.

[Z] H. Zassenhaus, Über endliche Fastkörper, Abh. Math. Sem. Univ. Hamburg 11 (1935), $187-220$.

Department of Mathematical Sciences, Northern Illinois Univeristy, DeKalb, ILLINOIS 60115 